\title{
Identification of Salmonella for Public Health Surveillance using Whole Genome Sequencing
}

Philip M Ashton, Satheesh Nair, Tansy M Peters, Janet Bale, David G Powell, Anaïs Painset, Rediat Tewolde, Ulf Schaefer, Claire Jenkins, Timothy J Dallman, Elizabeth M de Pinna, Kathie A Grant

In April 2015, Public Health England implemented whole genome sequencing (WGS) as a routine typing tool for public health surveillance of Salmonella, adopting a multilocus sequence typing (MLST) approach as a replacement for traditional serotyping. The WGS derived sequence type (ST) was compared to the phenotypic serotype for 6887 isolates of S. enterica subspecies I, and of these, $6616(96 \%)$ were concordant. Of the $4 \%(n=271)$ of isolates of subspecies I exhibiting a mismatch, 119 were due to a process error in the laboratory, 26 were likely caused by the serotype designation in the MLST database being incorrect and 126 occurred when two different serovars belonged to the same ST. The population structure of $S$. enterica subspecies II-IV differs markedly from that of subspecies I and, based on current data, defining the serovar from the clonal complex may be less appropriate for the classification of this group. Novel sequence types that were not present in the MLST database were identified in $8.6 \%$ of the total number of samples tested (including S. enterica subspecies I-IV and S. bongori) and these 654 isolates belonged to 326 novel STs. For S. enterica subspecies I, WGS MLST derived serotyping is a high throughput, accurate, robust, reliable typing method, well suited to routine public health surveillance. The combined output of ST and serovar supports the maintenance of traditional serovar nomenclature while providing additional insight on the true phylogenetic relationship between isolates. 


\section{Identification of Salmonella for public health surveillance using whole genome}

$$
\text { sequencing }
$$

3

4

5 Philip M. Ashton, Satheesh Nair, Tansy M. Peters, Janet Bale, Ulf Schaefer, David G. Powell, Anais

6 Painset, Rediat Tewolde, Claire Jenkins, Timothy J. Dallman*, Elizabeth M. de Pinna, Kathie A. Grant and

7 the Salmonella Whole Genome Sequencing Implementation Group

8

9 Gastrointestinal Bacteria Reference Unit, Public Health England, 61 Colindale Ave, London, NW9 5HT

12 Corresponding author

13 Timothy J. Dallman, Gastrointestinal Bacteria Reference Unit, Public Health England, 61 Colindale Ave,

14 London, NW9 5HT

15 Email: tim.dallman@phe.gov.uk

16

17

18

19

20

21 Running title: WGS derived MLST serotyping for Salmonella

22

23 


\section{Abstract}

26 In April 2015, Public Health England implemented whole genome sequencing (WGS) as a routine typing

27 tool for public health surveillance of Salmonella, adopting a multilocus sequence typing (MLST)

28 approach as a replacement for traditional serotyping. The WGS derived sequence type (ST) was

29 compared to the phenotypic serotype for 6887 isolates of $S$. enterica subspecies I, and of these, 6616

30 (96\%) were concordant. Of the $4 \%(n=271)$ of isolates of subspecies I exhibiting a mismatch, 119 were

31 due to a process error in the laboratory, 26 were likely caused by the serotype designation in the MLST

32 database being incorrect and 126 occurred when two different serovars belonged to the same ST. The

33 population structure of $S$. enterica subspecies II-IV differs markedly from that of subspecies I and, based

34 on current data, defining the serovar from the clonal complex may be less appropriate for the

35 classification of this group. Novel sequence types that were not present in the MLST database were

36 identified in $8.6 \%$ of the total number of samples tested (including $S$. enterica subspecies I-IV and $S$.

37 bongori) and these 654 isolates belonged to 326 novel STs. For S. enterica subspecies I, WGS MLST

38 derived serotyping is a high throughput, accurate, robust, reliable typing method, well suited to routine

39 public health surveillance. The combined output of ST and serovar supports the maintenance of

40 traditional serovar nomenclature while providing additional insight on the true phylogenetic relationship

41 between isolates. 


\section{Introduction}

44 The Salmonellae are major human pathogens and represent a significant global public health issue 45 causing morbidity and mortality resulting in a high social and economic burden worldwide (Majowicz et 46 al., 2010). The genus consists of 2 species; Salmonella enterica and S. bongori. There are six subspecies of S. enterica differentiated by biochemical variations, namely subspecies enterica (I), salamae (II), arizonae (IIla), diarizonae (IIIb), houtenae (IV) and indica (VI) (Threlfall et al. 1999). Subspecies I, S. enterica subsp. enterica cause $99 \%$ of human and animal infections. The two main pathologies associated with S. enterica are gastroenteritis and typhoidal disease. The typhoidal Salmonellae include

51 S. Typhi and S. Paratyphi A, B and C. They are host restricted, monophyletic, rarely undergo recombination events and exhibit convergent evolution driven by genome degradation (Wain et al. 2015). The majority of gastroenteritis in the UK is caused by the host generalist serovars, such as $S$. Typhimurium and $S$. Enteritidis, and host adapted serovars that are adapted to a specific animal reservoir but can infect man and include S. Dublin, S. Gallinarum S. Choleraesuis, and S. Bovismorbificans (Langridge et al. 2015).

57

Approximately 8,000 isolates are referred to the Salmonella Reference Service (SRS) at Public Health England (PHE) each year from local and regional hospital laboratories. In April 2015, PHE implemented whole genome sequencing (WGS) as the routine typing tool for public health surveillance of Salmonella infections. Prior to April 2015, presumptive Salmonella isolates referred to SRS were speciated and subspeciated using PCR (Hopkins et al. 2009, 2011) and grouped into serovars as described in the WhiteKauffman-Le Minor scheme (Grimont \& Weill 2007, Guibourdenche et al. 2010, Issenhuth-Jeanjean et al. 2014). This methodology is based on reactions of rabbit antisera to the lipopolysaccharide ( $O$ antigen encoded by $r f b$ genes) and flagellar antigens (phases 1 and 2 of $\mathrm{H}$ antigen encoded by fliC and fljB). The scheme utilises this phenotypic variation, expressed as an antigenic formulae, to divide Salmonella into more than 2600 serovars. Epidemiological investigations of Salmonella infecting humans and animals have relied on serotyping for over 70 years; national and international governmental agencies base guidelines and regulations on the serotyping method and the use of this nomenclature is a globally recognised form of communication (Swaminathan et al. 2009, EFSA 2010). Furthermore, serovars have

71 often been shown to correlate with host range and disease sequelae (Gordon et al. 2011, Wain et al. 72 2015, Langridge et al. 2015). 
74 There are, however, a number of issues with the serotyping approach; specifically, the expense and 75 expertise required to produce the antisera and, furthermore, serotyping does not reflect the genetic 76 relatedness between serovars, nor does it provide an evolutionary perspective. Alternative molecular 77 serotyping methods have been described previously including Pulsed-field gel electrophoresis, 78 ribotyping, repetitive extragenic palindromic sequence-based PCR (rep-PCR) and combined PCR- and 79 sequencing-based approach that directly targets $\mathrm{O}$ - and H-antigen-encoding genes (Ranieri et al. 2013, 80 Shi et al. 2015). In 2012, Achtman et al. proposed a sequenced based approach, multilocus sequence 81 typing (MLST), based on the sequences of multiple house-keeping genes. Isolates that possess identical 82 alleles for the seven gene fragments analysed are assigned a common sequence type (ST) and related 83 STs from clonal complexes are termed e-Burst Groups (eBGs). They showed that ST and eBGs strongly 84 correlated with serovar and so utilising this approach would facilitate backward compatibility with 85 historical data, minimise disruption for reference laboratory service users and facilitate data exchange 86 with other colleagues in the field.

Advances in whole genome sequencing (WGS) methodologies have resulted in the ability to perform high throughput sequencing of bacterial genomes at low cost making WGS an economically viable alternative to traditional typing methods for public health surveillance and outbreak detection (Koser et al. 2012). Whilst WGS provides the opportunity to resolve bacterial strains to the single nucleotide resolution needed for identifying cases linked to a common source of infection (Dallman et al. 2015), grouping isolates into higher taxonomical clones (e.g. those defined by serotyping) is an important step. The decision to adopt WGS as a routine typing method at PHE provided the opportunity to review our approach to typing Salmonella and to implement the MLST approach in parallel with WGS.

The aim of this study was to evaluate MLST, as derived from WGS data, as a replacement for conventional serotyping of Salmonella for routine public health surveillance and to provide insight into the genetic population structure of all Salmonella species in England and Wales during a 12 month period. 


\section{Bacterial strains}

107 All isolates $(n=7465)$ of Salmonella from human cases of gastrointestinal disease submitted to SRS from

108 local and regional hospital laboratories in England \& Wales between $1^{\text {st }}$ April 2014 and $31^{\text {st }}$ March 2015

109 were sequenced in parallel with phenotypic serotyping (Supplementary Table). Of these, 7338 were

110 identified as subspecies I and included 263 different serovars. The ten most common serovars in this

111 dataset were Enteriditis (2310), Typhimurium (1407), Infantis (184), Typhi (184), Newport (173), Virchow

112 (162), Kentucky (160), Stanley (146), Paratyphi A (135) and Java (99). One hundred and twenty seven

113 isolates were identified as subspecies II-IV (S. enterica subspecies salamae $n=28$; S. enterica subspecies

114 arizonae $n=25$; $S$. enterica subspecies diarizonae $n=49$; $S$. enterica subspecies houtenae $n=20$ ) and there

115 was one isolate of $S$. bongori. No isolates belonging to subspecies VI (S. enterica subspecies indica) were

116 submitted to SRS during the study period.

117

118 DNA extraction for WGS

119 DNA extraction of Salmonella isolates was carried out using a modified protocol of the Qiasymphony

120 DSP DNA midi kit (Qiagen). In brief, $0.7 \mathrm{ml}$ of overnight Salmonella culture in a 96 deep well plate was

121 harvested. Bacterial cells were pre-lysed in $220 \mu \mathrm{l}$ of ATL buffer (Qiagen) and $20 \mu \mathrm{l}$ Proteinase K

122 (Qiagen), and incubated shaking for 30 mins at $56^{\circ} \mathrm{C}$. Four $\mu$ of RNase at $100 \mathrm{mg} / \mathrm{ml}$ (Qiagen) was added

123 to the lysed cells and re-incubated for a further $15 \mathrm{mins}$ at $37^{\circ} \mathrm{C}$. This step increases the purity of the

124 DNA for further downstream sequencing. Extraction of DNA from the treated cells was performed on

125 the Qiasymphony SP platform (Qiagen) and eluted in $100 \mu$ of water. DNA concentration using the

126 GloMax system (Promega) was determined for the following sequencing steps.

127

128 DNA sequencing

129 Extracted DNA was then processed using the NexteraXT sample preparation method and sequenced

130 with a standard 2×101 base protocol on a HiSeq 2500 Instrument in fast mode (Illumina, San Diego).

131

\section{Bioinformatics Workflow}

133 FASTQ reads were quality trimmed using Trimomatic (Bolger et al. 2014) with bases removed from the

134 trailing end that fell below a PHRED score of 30 . If the read length post trimming was less than $50 \mathrm{bp}$ the

135 read and its pair were discarded. The PHE KmerID pipeline (https://github.com/phe-

136 bioinformatics/kmerid) was used to compare the sequenced reads with 1769 published genomes to

137 identify the bacterial species (and Salmonella subspecies) and to detect cultures submitted by the local 
138 and regional hospital laboratories that contained more than one bacterial species (mixed cultures).

139 KmerID determines a similarity index between the FASTQ reads and each of the 1769 published

140 reference genomes by calculating the percentage of 18-mers in the reference that are also present in

141 the FASTQs. Only 18-mers that occur at least twice in the FASTQ are considered present. Mixed cultures

142 are detected by comparing the list of similarities between the sample and the references with the

143 similarities of the references to each other, and filtering this comparison for inconsistencies. ST

144 assignment was performed using the Metric Orientated Sequence Typer (MOST), a modified version of

145 SRST (Inouye et al., 2012), available from https://github.com/phe-bioinformatics/MOST. The primary

146 difference between SRST and MOST is in the metrics provided around the result, while SRST gives a

147 single score, MOST provides a larger array of metrics to give users more details on the read level

148 associated with their result. Preliminary analysis was undertaken using the MLST database described in

149 Achtman et al. (2012). It takes approximately 10-15 minutes to run MOST using a single core on the PHE

150 infrastructure which consists of Intel Xeon CPU E5-2680 0 @ 2.70GHz, 16 cores sharing 125Gb Memory.

151

152 For isolates that had novel STs, or a ST but no associated serovar in the Achtman MLST database, the

153 serovar was determined by phenotypic serotyping at PHE. STs and corresponding serovars of isolates

154 serotyped and sequenced during this study were added to a modified version of the Achtman MLST

155 database, held and curated at PHE. These novel STs were assigned a preliminary ST (PST) and an

156 inferred serovar was determined. The PHE MLST database currently holds 7000 strains and 1,200

157 serovars and is up-dated every three months.

158

159 For some STs that contained two serotypes, whole genome SNP phylogenetic analysis was carried out by

160 mapping the strains of interest against a reference genome from within the same sequence type (for

161 ST909 H145100685 was used; for ST49, H143720759 was used), using BWA mem (Li \& Durbin, 2009).

162 SNPs were called using GATK2 (DePristo et al., 2011) in unified genotyper mode. Core genome positions

163 that had a high quality SNP ( $>90 \%$ consensus, minimum depth $10 x, G Q>=30, M Q>=30$ ) in at least one

164 strain were extracted and RAxML v8.1.17 phylogenies determined with the gamma model of rate

165 heterogeneity and 100 bootstraps undertaken.

166

167

168

169 Results 
170 Achtman et al. (2012) described the population structure of Salmonella enterica as monophyletic

171 lineages of STs that have evolved from a single founder node and termed these discrete clusters eBGs.

172 The population structure of all the Salmonella species submitted to PHE between April 2014 and March

1732015 is illustrated by the minimum spanning tree in Figure 1.

174

\section{Salmonella subspecies I}

176 The ST and corresponding serovar designation obtained from the MLST database were used to compare

177 the WGS derived ST to the phenotypic serotype for 6887 (94\%) of 7338 isolates of subspecies 1, and of

178 these, 6616 (96\%) had the same result by both methods (Supplementary Table). It was not possible to

179 compare phenotypic serotyping with MLST-based serotyping for 451 (6\%) subspecies I isolates because

180 either the phenotypic serotype could not be determined due to an incomplete antigenic structure ( $S$.

181 Unnamed) ( $n=423$ ) or the serovar could not be determined because the ST did not have a designated

182 serotype in the MLST database $(n=70)$. Forty-two isolates were both S. Unnamed and had no MLST

183 designated serotype.

184

185 For the $423(5.8 \%)$ subspecies I isolates reported as S. Unnamed, 318 (90\%) were designated a serotype

186 from the WGS derived MLST data. The most common serovars typed in this way included $S$.

187 Typhimurium (118), S. Virchow (30), S. Stanley (17), S. Enteritidis (16), S. Infantis (14) and S. Thompson

188 (13). Of the 7338 strains tested, 70 (1\%) had no serotype designation in the MLST database, of which 28

189 (40\%) were serotyped phenotypically (Supplementary Table).

190

191

Subspecies I novel sequence types

192 Novel sequence types that were not present in the MLST database were identified in $8.6 \%(n=654)$ of

193 the strains (Supplementary Table). These 654 isolates belonged to a total of 326 novel STs, designated

194 PST; the modal number of isolates identified per PST was one (Figure 2a). There was no difference in

195 the distribution of number of isolates per PST depending on whether the PST had a known serovar or

196 belonged to an unnamed or ambiguous serotype. The rate at which PSTs were received throughout the

197 year was plotted and revealed a linear relationship $\left(R^{2}=0.98, y=1.04 * x\right.$, where $x=$ number of days

198 since April $1^{\text {st }} 2014$ ) (Figure 2b).

199

200 The serovars with the highest number of new PSTs were S. Typhimurium (n=9), S. Stanley $(n=9), S$.

201 Enteritidis $(n=9)$ and $S$. Newport $(n=8)$, although the majority of these PSTs were single locus variants 
202 (SLVs) of established STs, belonging to these serovars (S. Typhimurium 8/9, S. Stanley 7/9 and S.

203 Newport 7/8). There were also serovars for which a large number of PSTs were identified that were not

204 SLVs of established STs (S. Agama 5/5, S. Agbeni 5/5, S. Saint-Paul 5/5, S. Enteritidis 4/9) which may

205 represent new eBGs that share these serotypes.

206

207 Subspecies I mismatches

208 Four percent $(n=271)$ of the isolates tested exhibited a mismatch between the WGS MLST derived 209 serovar and the phenotypic serotyping results. Of the 271 mismatches, 119 were due to a process error 210 in the laboratory either in the phenotypic serotyping or the DNA extraction part of the WGS pipeline.

211 With respect to the phenotypic serotyping, common errors included mislabelling samples and

212 misinterpreting or incorrectly transcribing the antigenic structure, especially when the antigenic

213 structures were similar. For example, S. Agona (I 4, 12:f, g, s:-) and S. Derby (I 4, 12:f, g:-). DNA

214 extraction errors were associated with mislabelled samples.

215

216 Twenty-six mismatches were potentially caused by the predicted serotype designation in the Achtman

217 MLST database being incorrect which may be attributed to single entries that had been misidentified at

218 the laboratory from which the MLST data was submitted. For example, in the original database ST1499

219 is represented by one entry identified by the submitter as S. Litchfield. Subsequently, phenotypic

220 serotyping at PHE identified this ST as S. Bovis-morbificans in more than five isolates. ST1499 belongs to

221 eBG34 which comprises two other STs both associated with S. Bovis-morbificans, indicating that the

222 original entry in the MLST database is likely to be incorrect.

223

224 The most common reason for mismatches occurring between the WGS MLST derived serotype and the 225 phenotypic serotype $(n=126)$ occurred when two different serovars belonged to the same eBG and the 226 same ST (see Table 1 and discussed in more detail below).

228 For 157 of the 271 mismatches, assembly based ST calls were available from Enterobase

229 (http://enterobase.warwick.ac.uk/). We compared the STs called by our mapping pipeline and the 230 assembly based Enterobase pipeline and the same ST was called in $100 \%$ of samples.

231

232

233

Serovars Enteritidis and Dublin 
234 Of the 2308 isolates of $S$. Enteritidis identified by both phenotypic serotyping and WGS MLST derived 235 serotyping, 2296 belonged to eBG4, including 2200 ST11 and 76 ST183 (Figure 1). There were five

236 additional SLVs of ST11, four of which were novel types. S. Gallinarum and S. Pulloram can be difficult to

237 distinguish from S. Enteritidis (Thomson et al. 2008) but neither of these serovars were identified in this

238 study. Serologically, S. Dublin ([1],9,12:g,p:-) has a similar antigenic structure to $S$. Enteritidis

239 ([1],9,12:g,m:-), and in Achtman et al. (2012), eBG32 (ST74) contained both S. Enteritidis and S. Dublin.

240 However, in this study both isolates belonging to ST74 eBG32 typed as S. Enteriditis. Of the 2308

241 isolates, 26 belonged to nine new PSTs. The most common was P3147, a previously undescribed SLV of

242 ST11, comprising 16 cases including 10 known to have travelled to Malaysia or Singapore.

243

244 Serovar Typhimurium

245 In this study, eBG1 contained 1392 isolates of $S$. Typhimurium and monophasic S. Typhimurium (rough

246 and non-motile variants) (Hopkins et al. 2012). The monophasic variants also belong to eBG138

247 (primarily ST 36) and eBG243. In contrast to eBG1 described in Achtman et al. (2012), which was

248 represented by a large central ST19 node with at least 27 SLV STs comprising much smaller numbers of

249 strains, eBG1 in the PHE dataset shows a predominance of both ST19 and ST34 and less allelic variation.

250 Only nine SLVs to ST19 were identified including three undesignated STs (Figure 1).

251

252 Serovars Java/Paratyphi B data

253 Despite the different disease outcomes associated with S. Paratyphi B (most commonly associated with 254 invasive disease and paratyphoid fever) and S. Java (most commonly associated with gastroenteritis) it is 255 not possible to differentiate the two serotypes by serotyping alone. S. Java and S. Paratyphi B are

256 therefore differentiated in the laboratory by their ability to ferment dextrorotatory tartrate (S. Java $d T a+$ 257 and S. Paratyphi B dTa-) (Malorny et al. 2003).

259 The 99 isolates identified by both phenotypic serotyping and WGS MLST derived serotyping as S. Java, 260 belonged to a diverse range of eBGs, STs and PSTs (Table 2 and Figure 1). Two of these 99 isolates

261 (marked with * in Table 2) belonged to ST86 and the predicted serotype from the MLST database was S.

262 Paratyphi B. One of these isolates was from a blood culture (associated with invasive disease) and, 263 therefore, likely to have been misidentified phenotypically. All 12 isolates identified as S. Paratyphi B 264 phenotypically, were identified as S. Paratyphi B ST86 by WGS MLST. 
266

267

268

269

270

271

272

273

274

275

276

277

278

279

280

281

282

283

284

285

286

287

288

289

290

291

292

293

294

295

296

297

Subspecies II-IV and S. bongori

Isolates from subspecies II, III, IV, VI and S. bongori were not well represented in Achtman's MLST database and thus the majority of isolates from these sub-species sequenced in this study did not belong to a previously designated eBG or ST. The population structure of the 127 non-subspecies I isolates differs markedly from that of Salmonella enterica (subspecies 1) (Figure 1) and shows some similarity to the population structure of lineage 3 in being a connected network of STs.

Sixteen of the 28 isolates belonging to subspecies II were previously designated S. Unnamed and the 28 strains belonged to 20 different STs. There were 25 isolates classed as subspecies Illa (belonging to 10 different STs) and 49 in subspecies IIIb (belonging to 27 different STs). Of the 20 isolates identified as subspecies IV, 10 were designated S. Wassenaar (P3029) by phenotypic serotyping and the 20 isolates belonged to five different STs. All isolates of subspecies II-IV and S. bongori were correctly speciated using the k-mer ID approach.

\section{Population structure}

As highlighted by Achtman et al. (2012), the majority of isolates in the dataset belong to eBGs that have a one-to-one relationship with a specific serovar including S. Typhi, S. Paratyphi A and S. Heidelberg. In this study, of the serovars comprising more than 25 isolates, there were 17 serovar specific eBGs and 10 examples of a single serovar being associated with multiple eBGs (Figure 1). There were at least six examples of more than one serovar belonging to the same eBG but different STs, for example S. Hadar (ST33) and S. Kottbus (ST582) both belong to eBG22 and S. Bredeney (ST306) and S. Schwarzengrund (ST96) both belong to eBG33 (Supplementary Table).

There were seven examples where two serovars belonged to the same eBG and the same ST (Table 1). In all of these examples, the antigenic structures of the two serovars were similar with only one antigen differentiating the two serovars. Further analysis was carried out on two examples to determine whether this difference in antigenic structure represented a true difference in strain relatedness or a random change that is not reflected in phylogeny (for example, the insertion of phage encoded antigen). The analysis showed that the change in antigenic structure in $S$. Richmond $(16,7: y: 1,2)$ and $S$. Bareilly $(I$ 6, 7:y:1,5), both ST 909, and in S. Saintpaul (I 4,5,12: e,h: 1,2) and S. Haifa (I 4,5,12: z,10: 1,2), both ST49, reflected a true phylogenetic difference (Figures $3 a$ and $3 b$ ). 
298 The same higher strata population structure referred to as lineage 3 for S. enterica subspecies I, as

299 described by Achtman et al. (2012), was observed in this dataset (Figure 4). Genomes of these

300 Salmonellae are in constant flux and homologous recombination among unrelated eBGs is frequent

301 (Achtman et al. 2012, Didelot et al. 2011). Serovars in this lineage mainly consists of multiple eBGs and

302 are polyphyletic by nature. Achtman et al. (2012) suggested that the population structure of lineage 3

303 does not comprise of independent startbursts, as observed with other serovars of subspecies I, but

304 rather a connected network (Figure 4). The five most common examples of this in the current study,

305 were S. Oranienburg, S. Montevideo, S. Chester, S. Poona and S. Bredeney (Figure 4 and Supplementary

306 Table). These five serovars are not represented in the top 10 serovars submitted to SRS during this

307 surveillance period.

308

309 K-mer identification

310 There were 249 cultures submitted to SRS by the local hospital and regional laboratories for Salmonella

311 typing that were a mix of Salmonella and non-Salmonella species. These were identified by the k-mer

312 identification step and included 138 Escherichia coli, 40 Morganella morganii, 11 Citrobacter species and

313 four Escherichia albertii.

314

\section{Discussion}

316 In their seminal 2012 paper Achtman and colleagues argued convincingly for replacing serotyping with a

317 MLST approach based on genetic population groupings for typing S. enterica (Achtman et al. 2012). The

318 key aspects of this approach that led PHE to adopt this strategy were (i) the robustness of the

319 population structure as defined by the natural eBG clusters (ii) the fact that eBG designation provides an

320 accurate representation of strain relatedness and (iii) that this approach lends itself to automation. At

321 the same time, it was necessary for PHE to maintain serovar nomenclature in order to facilitate data

322 exchange with other colleagues in the field and maintain backward compatibility with historical data. It

323 was suggested that by using the MLST approach to infer serovar, and by reporting both inferred serovar

324 and ST, it would be possible to utilise the advantages of both methods and implement a state-of-the-art

325 typing system while keeping disruption for reference laboratory service users to a minimum.

326

327 The PHE dataset of 6887 subspecies I isolates that were serotyped using both traditional phenotypic

328 methods and a derived serotype based on MLST data extracted from the genome during a 12 month

329 time frame, provided further evidence of the robustness of the ST/eBG approach to typing. The $96 \%$ 
330 concordance between the two techniques in a reference laboratory setting is evidence of the validity

331 and suitability of this approach. There were 451 isolates that had to be excluded from the comparison

332 because both types of data (phenotypic and genotypic) were not available. Of these, for $94 \%$ of the

333 isolates, it was the phenotypic serotype that could not be determined indicating that WGS MLST derived

334 serotyping is more robust.

335

336 The PHE dataset included single serovars associated with multiple eBG, for example S. Typhimurium and

337 S. Newport (Sangal et al. 2010, Achtman et al. 2012) and multiple serovars belonging to the same eBG

338 but with different STs, for example S. Java (ST43) and S. Paratyphi (ST86) both belong to eBG5 (Achtman

339 et al. 2012). In both these scenarios, the correct serovar was determined from the MLST WGS data and

340 the combination of serovar and ST/eBG provided insight into the true phylogenetic relationship between

341 isolates. This data clearly supports Achtman and colleagues argument that eBG and ST designation

342 provides a more accurate representation of strain relatedness than the traditional serovar designation.

343 The phenomenon of multiple serovars belonging to the same ST (for example S. Richmond/S. Bareilly

344 and S. Haifa/S. Saintpaul) was a rare but important example of serotyping providing a higher level of

345 strain discrimination within a ST. These strains could be differentiated in silico using a tool to infer

346 serovar from the genes that determine antigenic struture, such as seqsero (Zhang et al. 2015).

348 Despite the implementation of WGS, a limited phenotypic serotyping facility continues to be maintained

349 at PHE in order to serotype isolates that cannot be matched to a serovar; either because the ST in the

350 MLST database has no serovar designation or the ST is a novel type. Additionally, it ensures that we

351 maintain the ability to perform the standard reference method for serotyping Salmonella. The PHE MLST

352 database is regularly up-dated to include STs recently matched to a serotype by linking the ST to PHE

353 phenotypic serotyping data and novel PSTs. This approach was adopted because at the time of analysis,

354 the Achtman MLST database was not accepting submissions generated by WGS. There was no decrease

355 in the rate at which PSTs were observed during the 12 month study period and the majority of PSTs

356 were only sampled once in that time frame. Many PSTs were SLVs of known STs, indicating that we have

357 not yet sampled the full diversity of known eBGs. New PSTs, not part of any previously identified eBG,

358 were also observed and further diversity was found within S. enterica subspecies II-IV and the lineage 3

359 population. This suggests that there is a large amount of previously unidentified diversity within the

360 species Salmonellae associated with both domestically acquired and travel related gastrointestinal

361 disease in human cases resident in England and Wales. 
363 Isolates exhibiting monophasic properties that could not be fully serotyped phenotypically because they 364 had an incomplete antigenic structure were matched to a ST derived serotype. The monophasic variants 365 in this study mainly belonged to eBG1, eBG138 and eBG243 and previous studies have also shown that 366 monophasic variants of $S$. Typhimurium have emerged as a result of multiple independent genetic 367 events (Soyer et al. 2009, Switt et al. 2009, Tennant et al. 2010). Strains with monophasic properties are 368 reportable to European Centre for Disease Prevention and Control (ECDC) but cannot be determined 369 using the ST approach. Alternative strategies for determining monophasic characteristics by PCR are 370 available (Prendergast et al. 2013) and methods for extracting this information from the genome 371 sequencing data have been developed at PHE (Personal communication: Philip Ashton \& Anna Lewis, 372 publication in preparation).

373

374 In contrast to S. Typhurmurium, where ST could not be used to determine monophasic characteristics, in 375 this study ST was able to differentiate the complex relationship between S. Java (Hazard Group (HG) 2 376 organism) and S. Paratyphi B (HG3) with the latter belonging to either ST42 or ST86. If this ST 377 designation proves to be robust, MLST will facilitate the diagnosis of invasive disease and life 378 threatening paratyphoid fever.

379

The MLST derived serovar correlated well with the traditional serovar designation and demonstrated many advantages over traditional phenotypic serotyping. Monophasic strains with incomplete antigenic structures were accurately assigned to serotypes. Phenotypic serotyping errors, such as misinterpreting or incorrectly transcribing the antigenic structure, were avoided. Novel types were identified, confirmed and given a PST designation. Finally, this approach lends itself to automation and rapid, high-throughput processing.

Two main issues arose during the evaluation of the MLST approach: (i) a number of STs did not have a serovar designation in the MLST database (including subspecies II to IV) and (ii) the unexpectedly large number of novel STs identified. Traditional phenotypic serotyping was required to type these isolates and the MLST database was modified and up-dated to incorporate the new data. Clearly, as we move forward the PHE MLST database will be constantly evolving and this data will be shared with colleagues

392 in the field via existing MLST databases and their WGS compliant successors e.g. EnteroBase \& BIGSdb. 393 While it is difficult to draw conclusions based on our small sample size, MLST may not currently be an 
394 appropriate tool for the classification of Salmonella sub-species II-IV, due to the lack of a discrete

395 population structure of EBGs. However, non-subspecies I isolates which are mainly adapted to cold 396 blooded animals and/or reptiles contributed to less than $1.7 \%$ of the workload during the time frame of

397 the study. Although MLST approach is generally more discriminatory than serotyping, it does not always

398 provide the fine resolution required for public health surveillance. Further analysis based on single

399 nucleotide polymorphisms in the core genome compared to a type strain representing the most

400 common eBGs is performed for outbreak detection and investigation (Ashton et al. 2014).

401

402 In conclusion, serotyping inferred from 7-gene MLST results derived from WGS data is an accurate, 403 robust, reliable, high throughput typing method that is well suited to routine public health surveillance 404 of Salmonella. This approach supports the maintenance of traditional serovar nomenclature and 405 provides further insight on the true evolutionary relationship between isolates, as well as a framework 406 for fine level typing within eBGs for surveillance, outbreak detection and source attribution.

407

408

409 Acknowledgements

410 We would like to thank all the members of the Salmonella Whole Genome Sequencing Implementation

411 Group including Steve Connell, Anna Lewis, Andy Levy, Clare Maguire, Clare Wend-Hansen, Martin Day, 412 James Rogers, Siham Ibrahim, Arlene Barcenilla, Vineet Patel, Kiran Jayan, Anthony Underwood, 413 Catherine Arnold and lan Harrison.

414

415 Data Deposition

416 All data from the Salmonella surveillance project are deposited in the BioProject of the SRA

417 PRJNA248792. A per sample listing of the Sequence Read Archive accessions is available in 418 Supplementary table 1. 
420

421

422

423

424

425

426

427

428

429

430

431

432

433

434

435

436

437

438

439

440

441

442

443

444

445

446

447

448

449

450

451

452

453

454

455

456

457

458

459

460

461

462

463

464

465

466

\section{References}

Achtman M, Wain J, Weill FX, Nair S, Zhou Z, Sangal V, Krauland MG, Hale JL, Harbottle H, Uesbeck A, Dougan G, Harrison LH, Brisse S, S. Enterica MLST Study Group. 2012. Multilocus sequence typing as a replacement for serotyping in Salmonella enterica. PLoS Pathogens 8(6):e1002776.

Ashton PM, Peters T, Ameh L, McAleer R, Petrie S, Nair S, Muscat I, de Pinna E, Dallman T. 2015.

Whole Genome Sequencing for the Retrospective Investigation of an Outbreak of Salmonella

Typhimurium DT 8. PLoS Curr 10:7. doi:

10.1371/currents.outbreaks.2c05a47d292f376afc5a6fcdd8a7a3b6.

Bolger AM, Lohse M, Usadel B. 2015. Trimmomatic: a flexible trimmer for Illumina sequence data. Bioinformatics. 2014:1-7. doi:10.1093/bioinformatics/btu170.

Dallman TJ, Byrne L, Ashton PM, Cowley LA, Perry NT, Adak G, Petrovska L, Ellis RJ, Elson R, Underwood A, Green J, Hanage WP, Jenkins C, Grant K, Wain J. 2015. Whole-genome sequencing for national surveillance of Shiga Toxin-producing Escherichia coli O157. Clinical Infectious Diseases 61(3):305-12.

DePristo MA, Banks E, Poplin RE, et al. 2011. A framework for variation discovery and genotyping using next-generation DNA sequencing data. Nat Genet. 43(5):491-498.

Didelot X, Bowden R, Street T, Golubchik T, Spencer C, McVean G, Sangal V, Anjum MF, Achtman M, Falush D, Donnelly P. 2011. Recombination and population structure in Salmonella enterica. PLoS Pathogens 7:e1002191

European Food Safety Authority. 2010. Scientific opinion on monitoring and assessment of public health risk of "Salmonella Typhimurium" strains. EFSA Journal 8.

Grimont PAD, Weill FX. 2007. Antigenic Formulae of the Salmonella Serovars (ninth ed.)WHO Collaborating Center for Reference and Research on Salmonella, Institut Pasteur, Paris (2007) http://www.pasteur.fr/sante/clre/cadrecnr/salmoms/WKLM En.pdf

Gordon MA. 2011. Invasive nontyphoidal Salmonella disease: epidemiology, pathogenesis and diagnosis. Current Opinion Infectious Diseases 24:484-489.

Guibourdenche M, Roggentin P, Mikoleit M, Fields PI, Bockemuhl J, Grimont PA, Weill FX. 2010. Supplement 2003-2007 (No. 47) to the White-Kauffmann-Le Minor scheme. Research in Microbiology 161:26-29.

Hopkins KL, Lawson AJ, Connell S, Peters TM, de Pinna E. 2011. A novel real-time polymerase chain reaction for identification of Salmonella enterica subspecies enterica. Diagnostic Microbiology \& Infectious Disease 70(2):278-80.

Hopkins KL, Peters TM, Lawson AJ, Owen RJ. 2009. Rapid identification of Salmonella enterica subsp. arizonae and S. enterica subsp. diarizonae by real-time polymerase chain reaction. Diagnostic Microbiology \& Infectious Disease 64(4):452-4. 
467 Hopkins KL, de Pinna E, Wain J. 2012. Prevalence of Salmonella enterica serovar 4,[5],12:i:- in England and Wales, 2010. Euro Surveillance 17(37). pii: 20275

469

470

Inouye M, Conway TC, Zobel J, Holt KE. Short read sequence typing (SRST): multi-locus sequence types

471

472

473

474

475

476

477

478

479

480

481

482

483

484

485

486

487

488

489

from short reads. 2012. BMC Genomics;13(1):338. doi:10.1186/1471-2164-13-338.

Issenhuth-Jeanjean S, Roggentin P, Mikoleit M, Guibourdenche M, de Pinna E, Nair S, Fields PI, Weill FX. 2014. Supplement 2008-2010 (no. 48) to the White-Kauffmann-Le Minor scheme. Research in Microbiology 165(7):526-30.

Koser CU, Ellington MJ, Cartwright EJ, Gillespie SH, Brown NM, Farrington M, Holden MT, Dougan G, Bentley SD, Parkhill J, Peacock SJ. 2012. Routine use of microbial whole genome sequencing in diagnostic and public health microbiology. PLoS Pathogens 8:e1002824.

Langridge GC, Fookes M, Connor TR, Feltwell T, Feasey N, Parsons BN, Seth-Smith HM, Barquist L, Stedman A, Humphrey T, Wigley P, Peters SE, Maskell DJ, Corander J, Chabalgoity JA, Barrow P, Parkhill J, Dougan G, Thomson NR. 2015. Patterns of genome evolution that have accompanied host adaptation in Salmonella. Proceedings of the National Academy of Sciences USA 112(3):863-8.

Li H, Durbin R. 2009. Fast and accurate short read alignment with Burrows-Wheeler transform. Bioinformatics. 25(14):1754-60.

Majowicz SE, Musto J, Scallan E, Angulo FJ, Kirk M, O'Brien SJ, Jones TF, Fazil A, Hoekstra RM; International Collaboration on Enteric Disease 'Burden of Illness' Studies. 2010. The global burden of nontyphoidal Salmonella gastroenteritis. Clinical Infectious Diseases (6):882-9.

491

492

Malorny B, Bunge C, Helmuth R. 2003. Discrimination of d-tartrate-fermenting and -nonfermenting Salmonella enterica subsp. enterica isolates by genotypic and phenotypic methods. Journal of Clinical Microbiology 41(9):4292-7.

496

497

Prager R, Rabsch W, Streckel W, Voigt W, Tietze E, Tschäpe H. 2003. Molecular properties of

498

499 Salmonella enterica serotype paratyphi B distinguish between its systemic and its enteric pathovars. Journal of Clinical Microbiology 41(9):4270-8.

500

501

502

503

504

505

506

507

Prendergast DM, Hand D, Ni Ghallchóir E, McCabe E, Fanning S, Griffin M, Egan J, Gutierrez M. 2013. A multiplex real-time PCR assay for the identification and differentiation of Salmonella enterica serovar Typhimurium and monophasic serovar 4,[5],12:i:-. International Journal Food Microbiology 166(1):48-53. doi: 10.1016/j.ijfoodmicro.2013.05.031.

Ranieri ML, Shi C, Moreno Switt Al, den Bakker HC, Wiedmann M. 2013. Comparison of typing methods with a new procedure based on sequence characterization for Salmonella serovar prediction. Journal of Clinical Microbiology 51(6):1786-97. doi: 10.1128/JCM.03201-12.

Sangal V, Harbottle H, Mazzoni CJ, Helmuth R, Guerra B, Didelot X, Paglietti B, Rabsch W, Brisse S, 511 Weill FX, Roumagnac P, Achtman M. 2010. Evolution and population structure of Salmonella enterica serovar Newport. Journal of Bacteriology 192(24):6465-76. 
514 Shi C, Singh P, Ranieri ML, Wiedmann M, Moreno Switt Al. 2015. Molecular methods for serovar determination of Salmonella. Critical Reviews Microbiology 41(3):309-25. doi: 10.3109/1040841X.2013.837862.

517

Soyer Y, Moreno SA, Davis MA, Maurer J, McDonough PL, Schoonmaker-Bopp DJ, Dumas NB, Root T, Warnick LD, Grohn YT, Wiedmann M. 2009. Salmonella enterica serotype 4,5,12:i:-, an emerging Salmonella serotype that represents multiple distinct clones. Journal of Clinical Microbiology 47: 35463556.

522

Swaminathan B, Gerner-Smidt P, Ng LK, Lukinmaa S, Kam KM, Rolando S, Gutierrez EP, Binsztein N. 2006. Building PulseNet International: an interconnected system of laboratory networks to facilitate timely public health recognition and response to foodborne disease outbreaks and emerging foodborne diseases. Foodborne Pathogens and Disease 3:36-50

Switt Al, Soyer Y, Warnick LD, Wiedmann M. 2009. Emergence, distribution, and molecular and phenotypic characteristics of Salmonella enterica serotype 4,5,12:i:-. Foodborne Pathogens and Disease 6(4):407-15. doi: 10.1089/fpd.2008.0213.

Tennant SM, Diallo S, Levy H, Livio S, Sow SO, Tapia M, Fields PI, Mikoleit M, Tamboura B, Kotloff KL, Nataro JP, Galen JE, Levine MM. 2010. Identification by PCR of non-typhoidal Salmonella enterica serovars associated with invasive infections among febrile patients in Mali. PLOS Neglected Tropical Diseases 4: e621.

Thomson NR, Clayton DJ, Windhorst D, Vernikos G, Davidson S, Churcher C, Quail MA, Stevens M, Jones MA, Watson M, Barron A, Layton A, Pickard D, Kingsley RA, Bignell A, Clark L, Harris B, Ormond D, Abdellah Z, Brooks K, Cherevach I, Chillingworth T, Woodward J, Norberczak H, Lord A, Arrowsmith C, Jagels K, Moule S, Mungall K, Sanders M, Whitehead S, Chabalgoity JA, Maskell D, Humphrey T, Roberts M, Barrow PA, Dougan G, Parkhill J. 2008. Comparative genome analysis of Salmonella Enteritidis PT4 and Salmonella Gallinarum 287/91 provides insights into evolutionary and host adaptation pathways. Genome Research 18(10):1624-37.

544

Threlfall J, Ward L, Old D. 1999. Changing the nomenclature of Salmonella. Communicable Diseases

Wain J, Hendriksen RS, Mikoleit ML, Keddy KH, Ochiai RL. 2015. Typhoid fever. Lancet 385(9973):113645.

Zhang S, Yin Y, Jones MB' Zhang Z, Deatherage Kaiser BL, Dinsmore BA, Fitzgerald C, Fields PI, Deng X. 2015. Salmonella serotype determination utilizing high-throughput genome sequencing data. $J$ Clin Microbiol 53(5):1685-92. doi: 10.1128/JCM.00323-15. 


\section{Tables}

556 Table 1. Examples where two serovars belonged to the same eBG and the same ST

557 Table 2. S. Java isolates in this study belonged to a diverse range of eBGs and STs associated with S. Java

558 whereas S. Paratyphi B belonged to ST86 only 


\section{Figures}

560 Figure 1. Population structure of all Salmonella enterica isolates submitted to PHE from local and

561 regional hospital laboratories in England and Wales between April 2014 and March 2015 (see

562 Supplementary Table for details)

563

564 Figure 2a. Novel, preliminary STs (PST) and the modal number of isolates identified per PST

565

566 Figure $2 \mathrm{~b}$. The rate at which PSTs were identified throughout the time frame of the study

567

568 Figure 3a. Phylogenetic relationship of S. Richmond and S. Bareilly (ST909) (Figure 3a) and S. Saintpaul 569 and S. Haifa (ST49) (Figure 3b)

570

571 Figure 4. Serovars in lineage 3 mainly consist of multiple eBGs and are polyphyletic by nature 572 


\section{Table 1 (on next page)}

Examples where two serovars belonged to the same eBG and the same ST 


\begin{tabular}{|c|c|c|}
\hline Serotype & Antigenic structure & ST \\
\hline $\begin{array}{l}\text { Bareilly } \\
\text { Richmond }\end{array}$ & $\begin{array}{l}\text { I6,7:y: } 1,2 \\
\text { I 6,7:y: } 1,5\end{array}$ & 909 \\
\hline $\begin{array}{l}\text { Saintpaul } \\
\text { Haifa }\end{array}$ & $\begin{array}{l}\text { I 4,5,12: e,h: 1,2 } \\
\text { | 4,5,12: z,10: } 1,2\end{array}$ & 49 \\
\hline $\begin{array}{l}\text { Sandiego } \\
\text { Brandenburg }\end{array}$ & $\begin{array}{l}\text { | 4,12: I,v: e,n,z15 } \\
\text { | 4,12: e,h: e,n,z15 }\end{array}$ & 20 \\
\hline $\begin{array}{l}\text { Uganda } \\
\text { Sinstorf }\end{array}$ & $\begin{array}{l}\text { I 3, 10: I,z13: 1,5 } \\
\text { I 3, 10: I,v: } 1,5\end{array}$ & 684 \\
\hline $\begin{array}{l}\text { Agona } \\
\text { Essen }\end{array}$ & $\begin{array}{l}\text { | 4,12: f,g,s:- } \\
\text { | 4,12: f,g,m:- }\end{array}$ & 13 \\
\hline $\begin{array}{l}\text { Napoli } \\
\text { Zaiman }\end{array}$ & $\begin{array}{l}\text { I 1,9,12: I,z13: enx } \\
\text { । 1,9,12: I,v: enx }\end{array}$ & P3141 \\
\hline
\end{tabular}

1 


\section{Table 2 (on next page)}

Differences in ST between S. Java and S. Paratyphi B

S. Java isolates in this study belonged to a diverse range of eBGs and STs associated with $S$. Java whereas S. Paratyphi B belonged to ST86 only 


\begin{tabular}{|c|c|c|c|c|c|c|c|c|c|c|}
\hline & \multicolumn{5}{|c|}{ eBG5 } & eBG 9 & eBG59 & \multicolumn{2}{|c|}{ eBG32 } & eBG95 \\
\hline $\begin{array}{c}\text { Phenotypic } \\
\text { serovar }\end{array}$ & ST43 & ST149 & ST307 & ST1577 & ST86 & ST88/127 & ST28 & ST423 & ST682/1588 & 1583 \\
\hline Java & 45 & 7 & 4 & 3 & $2 *$ & 18 & 6 & 5 & 2 & 1 \\
\hline Paratyphi B & 0 & 0 & 0 & 0 & 12 & 0 & 0 & 0 & 0 & 0 \\
\hline
\end{tabular}


Figure 1 (on next page)

Population Structure of Salmonella enterica submitted to PHE

Population structure of all Salmonella enterica isolates submitted to PHE from local and regional hospital laboratories in England and Wales between April 2014 and March 2015 (see Supplementary Table for details) 
Top 11 Salmonella

Lineage 3

Javiana

Chester

Oranienburg

Montevideo

Poona

Bredeney

\section{Kentucky}

Java
PEu'iv' - v

Subsp IV

Subsp II

Subsp IIla

Subsp IIIIb

Subsp V

Paratyphi B

Paratyphi A

Newport

Stanley

Virchow

Typhi

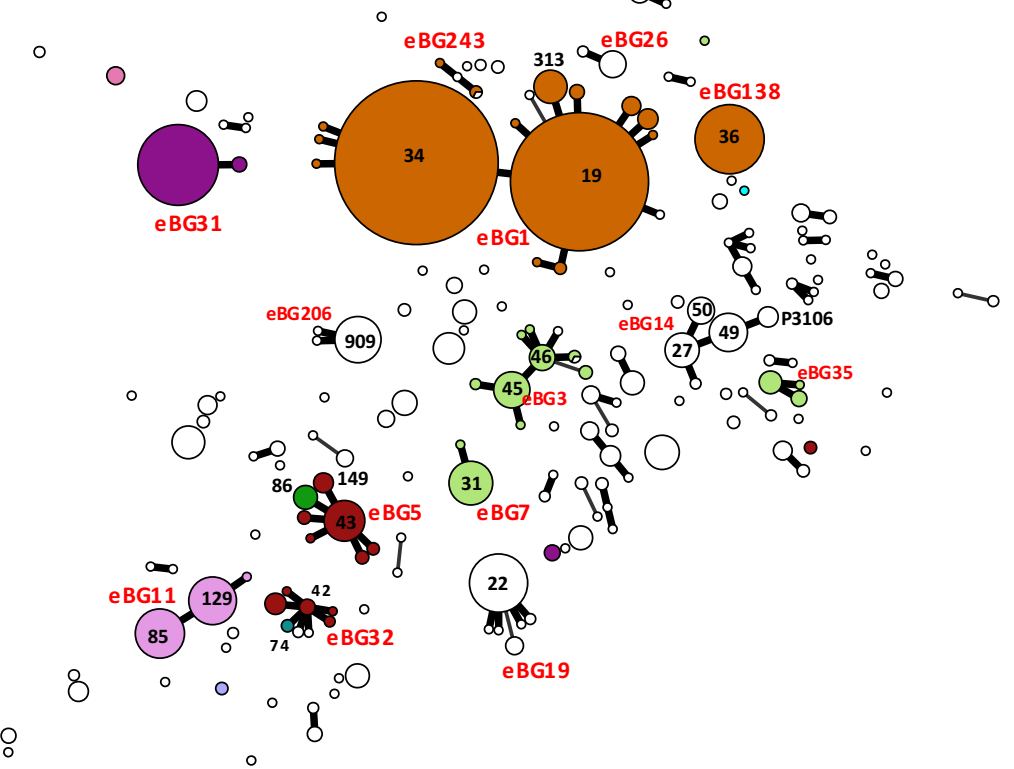


2

Trends in Preliminary Sequence Types

(A) Novel, preliminary STs (PST) and the modal number of isolates identified per PST (B) The rate at which PSTs were identified throughout the time frame of the study. 

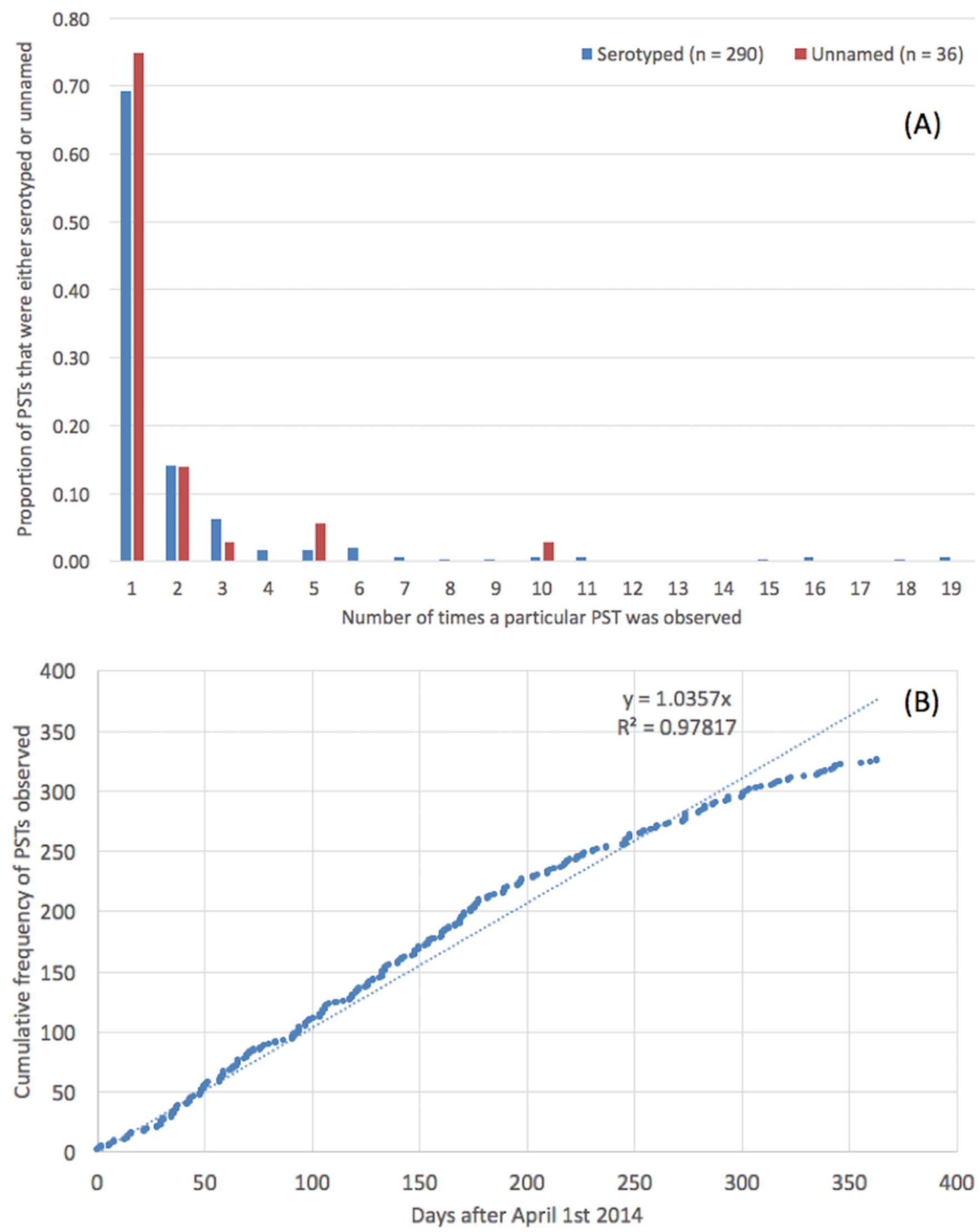
3

Phylogenetic relationship within two sequence types containing multiple serotypes

(A) Phylogenetic relationship of S. Richmond and S. Bareilly (ST909) (B) Phylogenetic relationship of S. Saintpaul and S. Haifa (ST49)
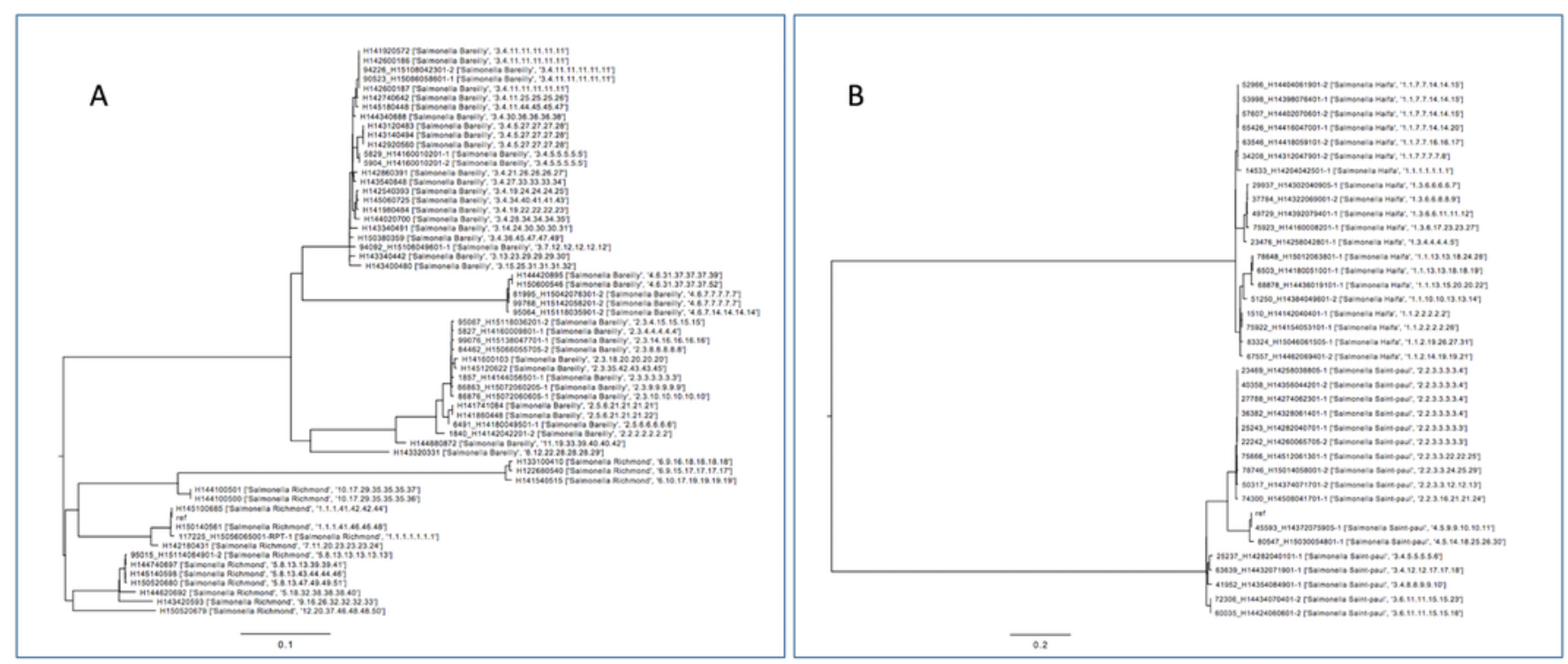
4

Salmonella lineage 3 population structure

Serovars in lineage 3 mainly consist of multiple eBGs and are polyphyletic by nature

Javiana - eBG17 (ST24,1674,437,P3211,P3212)

Chester - eBG49 (ST411,343,P3172), unassigned eBG(P3170)

Oranienburg - eBG44(ST1515,174,169), unassigned eBG(P3225) eBG203 (ST1512,1392,1576), unassigned eBG (ST864) eBG41 (ST23,47), unassigned eBG(P3216)

Montevideo - eBG40(ST4,699,195,81,316) eBG39(ST1518,138) Unassigned eBG (P3174)

Poona - eBG46 $(812,447)$

Unassigned eBG(714,P3210

Unassigned eBG(P3388,P3019,308,P3020,1069)

Bredeney - eBG240 (1596), eBG119 (505) eBG33 (306)
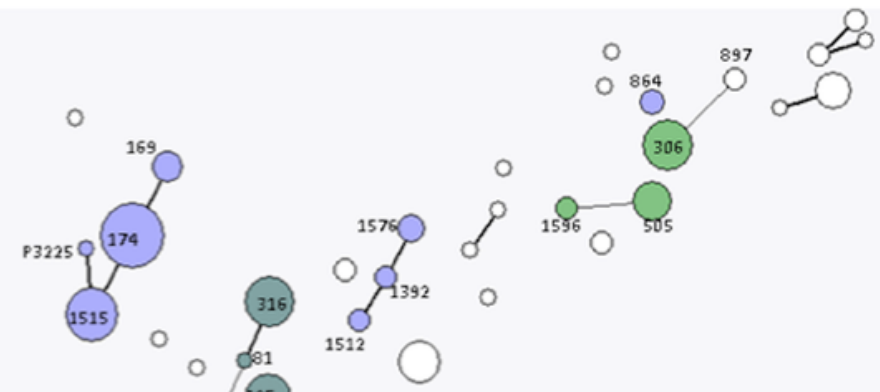

d

(1)<smiles>C1CC2CCC(C2)O1</smiles>

0

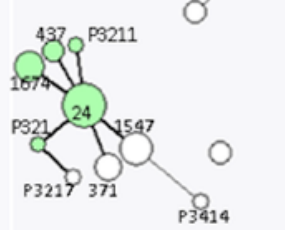

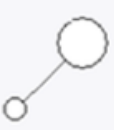

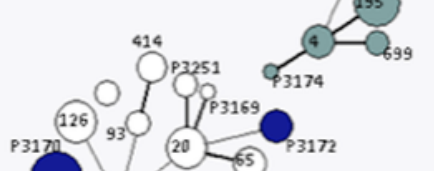

72
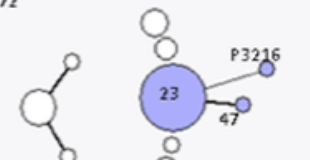

P3210
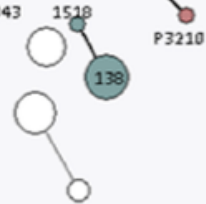

(33383

0

$\circ$ 